\title{
INFLUENCIA DE LA OSMOCONGELACIÓN SOBRE ALGUNAS PROPIEDADES FíSICAS DE PAPAYA (Carica papaya L.)
}

\section{INFLUENCE OF OSMODEHYDROFREEZING ON SOME PHYSICAL PROPERTIES OF PAPAYA (Carica papaya L.)}

\begin{abstract}
Alfredo Ayala Aponte ${ }^{1}$, Martha Sánchez ${ }^{2}$, Hanny Rodríguez ${ }^{3}$
${ }^{1}$ Doctor en Ciencia y Tecnología en Alimentos. Profesor Escuela de Ingeniería de Alimentos, Universidad del Valle, CaliColombia, e-mail: alfredo.ayala@correounivalle.edu.co; ${ }^{2}$ Ingeniera de Alimentos. Estudiante de Maestría en Ingeniería de Alimentos, Universidad del Valle-Cali, e-mail: misanchezt@gmail.com; ${ }^{3}$ Ingeniera de Alimentos. Universidad del Valle-Cali, e-mail: hannyvre@gmail.com
\end{abstract}

Rev. U.D.C.A Act. \& Div. Cient. 17(2): 487-494, Julio-Diciembre, 2014

RESUMEN

La osmo-congelación (OC) es el proceso combinado de aplicar la deshidratación osmótica (DO) seguido de la congelación en un alimento, para mejorar la calidad del producto congelado-descongelado. El objetivo de este trabajo fue evaluar la influencia de la aplicación de pretratamientos osmóticos sobre la pérdida de fase líquida (PFL), volumen (V) y color, en términos de claridad $\left(\mathrm{L}^{*}\right)$, tono $\left(\mathrm{h}^{\circ}\right)$ y cambio total de color $(\Delta \mathrm{E})$, en muestras de papaya, almacenadas en congelación. Las muestras fueron deshidratadas osmóticamente hasta 30 y 90 min, alcanzando, respectivamente, contenidos de humedad, de $81,40 \pm 0,69 \%$ y $76,24 \pm 0,41 \%$ (b.h); para ello, se empleó una solución osmótica (SO) de sacarosa, con $65^{\circ}$ Brix, a $26,0 \pm 0,2^{\circ} \mathrm{C}$. Las muestras deshidratadas osmóticamente fueron congeladas a $-40^{\circ} \mathrm{C}$ y, posteriormente, se almacenaron a $-18^{\circ} \mathrm{C}$, durante $10,20,30$ y 40 días. Como tratamiento control, se utilizaron muestras no tratadas osmóticamente (MNT), durante el almacenamiento en congelación. Los resultados mostraron que, en todos los tratamientos, el tiempo de almacenamiento en congelación influyó significativamente $(\mathrm{p}<0.05)$ sobre la pérdida de calidad de la fruta; sin embargo, las muestras DO a 90 min (DO6590) exhibieron las menores pérdidas de calidad, en términos de menor PFL, $\mathrm{V}$ y color, mientras que las muestra a 30 min (DO6530) mostraron las mayores pérdidas de PFL y V, pero con menores pérdidas de color respecto a las MNT. De acuerdo a estos resultados, se evidenció un efecto crioprotector de la técnica de OC sobre las muestras de papaya, principalmente, con DO hasta 76,24 $\pm 0,41 \%$ (b.h).

Palabras clave: Congelación, deshidratación osmótica, fase líquida, color.

\section{SUMMARY}

The osmo-dehydro-freezing $(\mathrm{OC})$ is the combined process of applying osmotic dehydration (OD) followed by freezing of a food product to improve the quality of frozen-thawed products. The aim of this study was to evaluate the influence of the application of osmotic pretreatments on the loss of liquid phase (PFL), volume (V) and color in terms of lightness $\left(\mathrm{L}^{*}\right)$, hue $\left(\mathrm{h}^{\circ}\right)$, and color change $(\Delta \mathrm{E})$ of papaya frozen stores samples. The osmotically dehydrated samples were treated during 30 and $90 \mathrm{~min}$, reaching moisture content of $81,40 \pm 0,69$ and $76,24 \pm 0,41 \%$ (wb) respectively. For this, an osmotic solution of sucrose with $65^{\circ}$ Brix at $26.0^{\circ} \mathrm{C} \pm 0.2$ was used. The treated samples were frozen at $-40^{\circ} \mathrm{C}$ and subsequently stored at $-18^{\circ} \mathrm{C}$ for $10,20,30$ and 40 days. As control osmotically untreated samples (MNT) during frozen storage were used. The results showed that in all treatments the frozen storage time significantly influenced $(p<0.05)$ the loss of fruit quality. However, the samples treated during 90 min (DO6590) showed the least loss of quality in terms of lower PFL, V and color; while the treated samples at 30 min (DO6530) showed the greatest loss of PFL and V; however presented lower losses in color compared with MNT. According to these results a cryoprotective effect of osmofreezing technique on samples of papaya was observed, mainly for samples with DO $76.24 \pm 0.41 \%$ (wb).

Key words: Freezing, osmotic dehydration, liquid phase, color. 


\section{INTRODUCCIÓN}

La papaya es una fruta de agradable sabor y aroma, que se cultiva ampliamente en los países tropicales y subtropicales. Se emplea en la elaboración de jugos, de pulpas, de mermeladas, de almíbar, de confitados y de otros productos (Kurozawa et al. 2014). Es fuente de un alto contenido de nutrientes (vitamina $\mathrm{C}$, provitamina $\mathrm{A}$ (carotenoides), entre otros) y minerales, como hierro, calcio potasio y sodio (Wall, 2006). La papaya es un fruto altamente perecedero por su alto contenido de humedad (CH), entre 80 y $85 \%$ (bh) (Ocoró \& Ayala, 2013), por lo que es importante encontrar alternativas para su conservación. La congelación es un método de conservación que permite prolongar la vida útil de los alimentos, manteniendo significativamente las características de calidad del producto (James et al. 2014); sin embargo, la congelación no es adecuada para todos los alimentos, en algunos casos, presenta cambios fisicoquímicos indeseables relacionados con la pérdida de calidad, una vez descongelado el producto (James et al. 2014). De acuerdo con Marani et al. (2007), la congelación puede provocar pérdidas significativas en la fase líquida y el color de los alimentos. Talens et al. (2001) manifiestan que los alimentos al descongelarse pueden perder la turgencia celular provocando flacidez, encogimiento y pérdida de textura. Tregunno \& Goff, (1996) explicaron que la disminución de la calidad en los alimentos congelados son debidos, principalmente, a que los cristales de hielo formados ocasionan daños irreversibles a los compartimentos celulares, asociados a la pérdida del estado osmótico y de la semi-permeabilidad.

Muchos procesos de congelación innovadores están siendo investigados y desarrollados en la actualidad, entre ellos, la osmocongelación (OC) (James et al. 2014; Bermúdez et al. 2013). La OC es un complemento de la congelación, donde el producto, primero, se deshidrata osmóticamente, a un nivel de humedad deseado y, después, se congela (Tregunno \& Goff, 1996). Su fundamento, se basa en la reducción del contenido de agua congelable, que significa disminución de cantidad de cristales de hielo formados durante la congelación (Talens et al. 2003).

Con la aplicación de la deshidratación osmótica (DO), se puede lograr reducir, parcialmente, una cantidad de agua congelable del producto. Este método consiste en la extracción de agua de un alimento que está sumergido en una solución osmótica (SO) hipertónica, por un tiempo y temperatura específicos (Ayala et al. 2009). La SO debe ser altamente concentrada de solutos, que pueden ser sal o azúcar y a temperatura ambiente (Marani et al. 2007).

La OC ha sido aplicada en diversas frutas y hortalizas con resultados positivos, después del proceso de descongelación, como en manzanas (Marani et al. 2007), kiwis (Talens et al.
2001), peras (Marani et al. 2007) y melón (Bermúdez et al. 2013). En papaya, se evidencia una investigación disponible en la literatura científica (Moyano et al. 2002); sin embargo, tanto la especie evaluada, Carica candamarcencis Hook f., como las condiciones de proceso son distintas a las de este trabajo, donde no se valoran diversos tiempos de almacenamiento en congelación.

Por consiguiente, el propósito de este trabajo fue evaluar la influencia de la aplicación de pretratamientos osmóticos sobre la pérdida de fase líquida (PFL), volumen (V) y color en términos de claridad $\left(\mathrm{L}^{*}\right)$, tono $\left(\mathrm{h}^{\circ}\right)$ y cambio total de color $(\Delta \mathrm{E})$ en muestras de papaya almacenadas en congelación durante 10, 20, 40 y 60 días.

\section{MATERIALES Y MÉTODOS}

Preparación de las muestras: Se utilizaron papayas (Carica papaya $L$.) de la variedad Maradol, con un estado de madurez homogéneo, con $13,5 \pm 0,8^{\circ}$ Brix y humedad de $87,2 \pm 0,4 \%$ (b.h.). Las frutas fueron lavadas, peladas (usando un cuchillo de acero inoxidable) y cortadas en dos partes, para remover las semillas. Del centro de cada parte de la fruta, se cortaron muestras de $20 \mathrm{~mm}$ de altura y $15 \mathrm{~mm}$ de diámetro, empleando un sacabocado cilíndrico en acero inoxidable.

Deshidratación osmótica: Se prepararon, aproximadamente, $5.000 \mathrm{~mL}$ de solución osmótica (SO) de sacarosa grado alimentario en agua destilada, con una concentración de $65^{\circ}$ Brix. Las muestras de papaya cortadas, se sumergieron en la SO de sacarosa, contenida en un recipiente de plástico. La relación fruta-SO fue de 1:20, para evitar cambios en la concentración de la SO (Falade \& Igbeka, 2007; Mayor et al. 2007; Rózek et al. 2009) y evitar una reducción de la fuerza impulsora durante el proceso (Ayşe \& İnci, 2009). Se empleó sacarosa, como soluto, debido a su amplio uso en el proceso de deshidratación de frutas (Osorio et al. 2007). La SO se mantuvo a $26,0 \pm 0,2^{\circ} \mathrm{C}$ y se agitó, permanentemente, a 800rpm, mediante un agitador mecánico (Kika Labor Technik Pol Col, US), para evitar el fenómeno de encostramiento por efecto del azúcar en la superficie de las muestras. En dos tiempos de DO (30 y 90min), las muestras se retiraron de la SO, alcanzando dos niveles de $\mathrm{CH}$, de 81,40 $\pm 0,69$ y $76,24 \pm 0,41 \%$ (b.h), respectivamente; con estos niveles de $\mathrm{CH}$, se reduce, parciamente, el agua congelable de la papaya. Las muestras DO al retirarse de la SO, se colocaron en papel (toallas) húmedo, para eliminar la solución remanente en la superficie.

A las muestras no tratadas (MNT) y tratadas osmóticamente (DO6530 y DO6590), se les determinaron el $\mathrm{CH}$, mediante el método 934.06 de la AOAC (1990) y los sólidos solubles ('Brix), mediante un refractómetro (Abbe Atago, Zeiss, USA), termostato a $20^{\circ} \mathrm{C}$. 
Congelación, almacenamiento y descongelación: Las MNT y tratadas osmóticamente, se almacenaron en una nevera comercial a $8^{\circ} \mathrm{C}$, en bolsas plásticas resellables, durante $12 \mathrm{~h}$, para promover el equilibrio interno de la concentración (Talens et al. 2001; Chiralt et al. 2001). Posteriormente, las muestras se congelaron a $-40^{\circ} \mathrm{C}$, en un congelador (Revco, (USA), a una velocidad de $1,1^{\circ} \mathrm{C} /$ min y se almacenaron en un congelador comercial a $-18^{\circ} \mathrm{C}$, durante $10,20,40$ y 60 días. Para cada tiempo de almacenamiento, las muestras se descongelaron a $8^{\circ} \mathrm{C}$, en una nevera comercial durante $18 \mathrm{~h}$, para evitar la presencia de hielo (Talens et al. 2003; Moraga et al. 2006; Sriwimon \& Boonsupthip, 2011). Una vez descongeladas las muestras, se evaluaron las propiedades físicas PFL, $V$ y color, en términos de $L^{*}, h^{*}$ y $\Delta \mathrm{E}$.

Determinación de propiedades físicas: La PFL, se determinó mediante la ecuación 1, la cual, evalúa las diferencias de peso de las muestras antes y después del proceso de congelación-descongelación (Talens et al. 2001; Sriwimon \& Boonsupthip, 2011) y, para ello, se utilizó una balanza analítica (Mettler Toledo AE200), con precisión 0,001g.

$$
P F L=\frac{m_{f}-m_{o}}{m_{o}}
$$

Donde: $\mathrm{m}_{\mathrm{o}}=$ peso de la muestra antes de congelar y $\mathrm{m} \mathrm{f}=$ peso de la muestra después de congelar-descongelar.

Para el volumen de las muestras de papaya, antes y después de la congelación-descongelación, se midió el diámetro y la altura en tres puntos separados, $120^{\circ}$, en una de las caras circulares del cilindro; para ello, se empleó un calibrador digital (Bull Tols, USA). Con base en estas mediciones, se determinó el cambio o pérdida de volumen $(\Delta \mathrm{V})$, mediante la ecuación 2.

$$
\Delta V=\frac{V f-V i}{V i}
$$

Donde $V_{f}$ y $V_{i}$ son respectivamente el volumen de muestra antes y después de la congelación-descongelación.

El color de las muestras de papaya, se midió a través del espectro reflexión, entre 400-700nm, mediante un espectrocolorímetro (Hunterlab Reston, USA). Se obtuvieron las coordenadas de color CIE-L*a*b* a partir de los espectros de reflexión de las muestras, utilizando, como referencia, el iluminante D65 y el observador $10^{\circ}$. Donde $L^{*}$ es Luminosidad o claridad, a* es coloración entre rojo-verde y b* coloración entre amarillo-azul. A partir de estas coordenadas, se estimaron $\mathrm{h}{ }^{\mathrm{ab}}$, y $\Delta \mathrm{E}$, mediante las ecuaciones 3 y 4 , respectivamente.

$$
\begin{gathered}
h^{*}{ }_{a b}=\operatorname{arctg} \frac{b^{*}}{a^{*}} \\
\Delta E=\sqrt{\Delta L^{*^{2}}+\Delta a^{*^{2}}+\Delta b^{*^{2}}}
\end{gathered}
$$

Diseño experimental: Se aplicó un diseño factorial $3 \times 4$ completamente al azar, con dos factores: el factor $\mathrm{CH}$ de la fruta, con tres niveles: $87,2 \pm 0,4 \%$ (MNT, fresco); $81,40 \pm 0,69 \%$ (DO6530) y 76,24 $\pm 0,41 \%$ (DO65 90) y el factor tiempo de almacenamiento en congelación, con 4 niveles: 10, 20, 40 y 60 días; los experimentos se realizaron por triplicado. Los resultados, se analizaron mediante análisis de varianza (ANOVA), con nivel de confiabilidad de 95\%; para ello, se empleó el programa Minitab 16 (Minitab, Inc., State College, Pensylvania, 2009).

\section{RESULTADOS Y DISCUSIÓN}

La figura 1 muestra la PFL de las MNT y muestras tratadas (DO6530 y DO6090), a distintos tiempos de almacenamiento en congelación. El tiempo de almacenamiento influyó significativamente $(p<0.05)$ sobre la PFL, en todos los tratamientos, incrementándose la pérdida durante el almacenamiento. Estos resultados están de acuerdo con los reportados en melón (Ayala et al. 2014), en fresa (Moraga et al. 2006) y en kiwi (Talens et al. 2001). Este incremento de PFL puede ser atribuido al proceso de recristalización del hielo durante el almacenamiento en congelación, ocasionando pérdida del contenido celular y pérdida de la capacidad de retención de agua de la célula (Goncalves et al. 2011). De acuerdo con Simandjuntak et al. (1996), el incremento de la PFL indica gran disminución de componentes líquidos celulares y puede ser causado por rompimiento mecánico de las membranas durante el almacenamiento en congelación.

Se puede notar que las muestras tratadas con menor nivel de humedad (DO6590) presentaron las menores PFL, por efecto de la congelación-descongelación, en los diferentes tiempos de almacenamiento. Este comportamiento puede ser debido a una menor recristalización del hielo por contener menor agua congelable y, en consecuencia, menor daño estructural. Otros investigadores han reportado similar comportamiento en diferentes frutas y vegetales (Marani et al. 2007) y en melón (Ayala et al. 2014; Bermúdez et al. 2013). Este resultado indica el efecto crioprotector de la técnica de $\mathrm{OC}$, al deshidratar osmóticamente la fruta, hasta un $\mathrm{CH}$ de $76,2 \%$ (b.h).

Comportamiento contrario presentaron las muestras tratadas osmóticamente a 30 min (DO6530), mostrando las mayores pérdidas de PFL, en todos los tiempos de almacenamiento. Este resultado contradice el presunto efecto crio- 


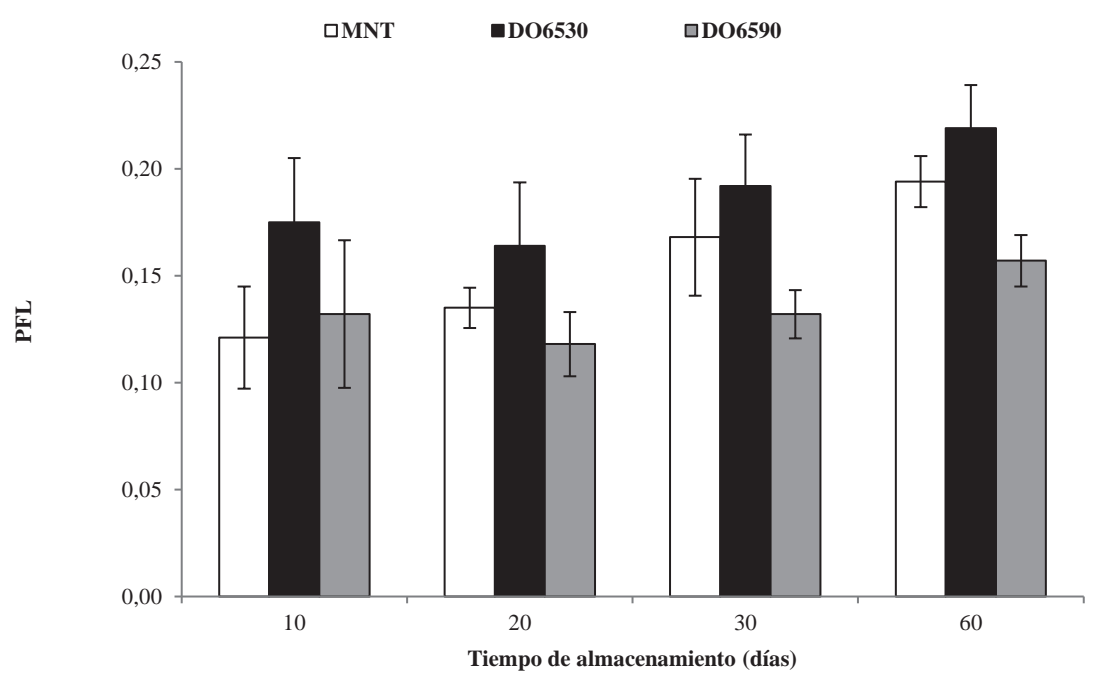

Figura 1. Pérdida de fase líquida en muestras de papaya tratadas y no tratadas, durante el proceso de congelación-descongelación.

protector de la DO previa a la congelación; resultado similar se presentó en melón (Bianchi et al. 2011) y en fresa (Marani et al. 2007.) Una posible explicación de este resultado puede estar atribuido a que el nivel de humedad $(81,40 \pm 0,69)$ alcanzado en el corto tiempo de DO (30 min) es suficiente para formar grandes cristales de hielo durante la congelación y, por tanto, dañar la estructura celular, ocasionado mayor PFL; de acuerdo a Marani et al. (2007), al incrementarse el tiempo de DO permite una disminución de la PFL. El ANOVA evidenció también un efecto significativo $(p<0.05)$ del factor nivel de humedad sobre la PFL de muestras de papaya.

En la figura 2, se muestran las pérdidas de volumen $(\Delta \mathrm{V}$ en fracción volumétrica) de las MNT y muestras tratadas osmóticamente (DO6530 Y DO6090), durante el almacenamiento. Se evidenció que el tiempo de almacenamiento en congelación influyó significativamente $(\mathrm{p}<0,05)$ sobre la $\Delta \mathrm{V}$ de la fruta, mostrando, en todos los tratamientos, mayores $\Delta \mathrm{V}$, durante el almacenamiento. Este resultado, se podría explicar por las PFL que revelaron las muestras en todos los tratamientos, durante el proceso de congelación-descongelación, explicado anteriormente. De acuerdo con Koç et al. (2008), las pérdidas de agua en un alimento provocan daños en la estructura, generando encogimiento y cambios en su microestructura. Por otro lado, el nivel de humedad influyó significativamente $(\mathrm{p}<0,05)$ sobre $\Delta \mathrm{V}$, mostrando que el tratamiento DO6590 mostró el menor cambio de volumen; resultado similar, se evidenció en la osmocongelación de melón (Bermúdez et al. 2013). Este comportamiento puede estar asociado con las menores PFL en el proceso de congelación-descongelación, debido a la menor cantidad de agua congelable, mientras que el tratamiento DO6530 presentó la mayor pérdida de vo- lumen en todos los tiempos de almacenamiento, alcanzando la mayor pérdida a los 60 días, con $35,9 \%$, posiblemente, a las mayores pérdidas de fase líquida, que pueden ocasionar mayor daño estructural y, en efecto, mayor encogimiento (Koç et al. 2008).

La figura 3 muestra la evolución de la claridad ( $L *)$ durante la osmocongelación de muestras de papaya. Se puede advertir, en todos los tratamientos, la disminución de $L^{*}$, por efecto del almacenamiento $(p<0,05)$, siendo las MNT las que evidenciaron la mayor variación desde el tiempo cero (no congeladas), con $42,51 \pm 0,71$ hasta el día 60 , con $27,85 \pm 1,21$, mientras que los tratamientos D06530 y D06590 variaron, respectivamente, desde $36,59 \pm 4,81$ hasta $26,82 \pm 2,82$ y desde $36,97 \pm 3,33$ hasta $27,85 \pm 3,57$. Estos resultados significan que las muestras disminuyeron la claridad durante el tiempo de almacenamiento en congelación, probablemente, a que el líquido celular liberado durante la PFL recubre la superficie del tejido de la muestra, ocasionado aumento de la transparencia y, en consecuencia, disminución de L*. De acuerdo con Talens et al. (2001), la transparencia se debe a un aumento de la homogeneidad del índice de refracción en el tejido celular; cuanto mayor es la transparencia más oscura es la muestra.

Al comparar L* entre los tres tratamientos durante el almacenamiento en congelación no se evidenciaron diferencias significativas ( $p>0,05)$, lo cual, indica que, independientemente del nivel de humedad o de tratamiento osmótico, la pérdida de L* es significativamente similar en la muestras de papaya, durante el almacenamiento congelado. 


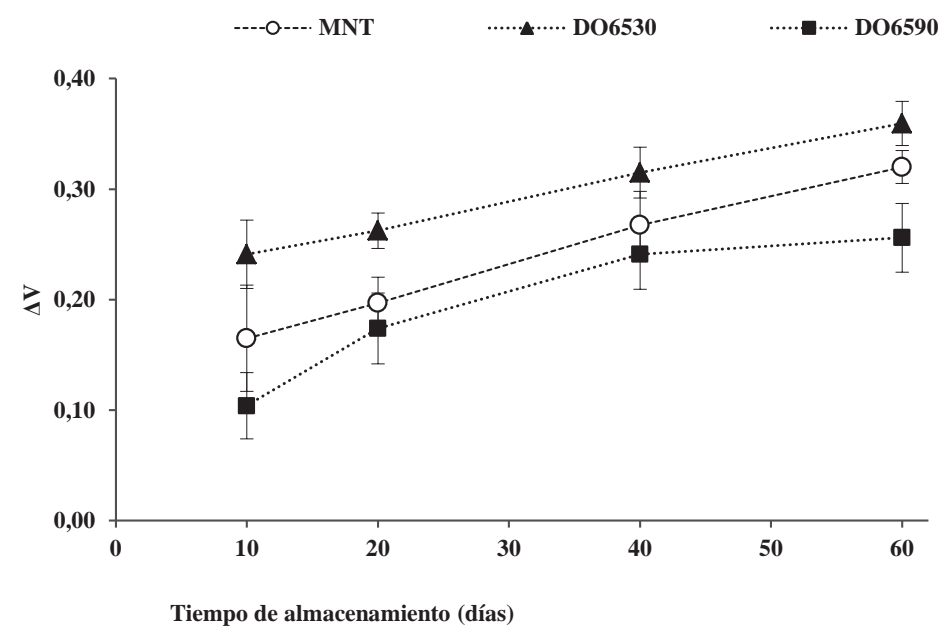

Figura 2. Pérdidas de volumen en muestras de papaya tratadas y no tratadas osmóticamente, durante el almacenamiento en congelación.

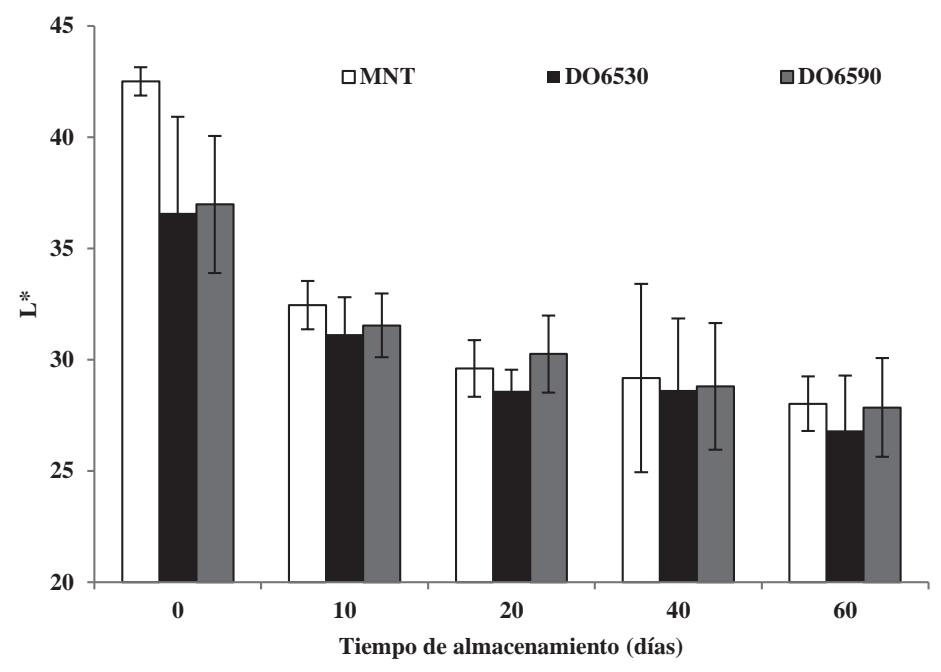

Figura 3. Cambio de claridad en muestras de papaya tratadas y no tratadas osmóticamente, durante el almacenamiento en congelación.

La figura 4 presenta la evolución del tono $\left(\mathrm{h}^{\circ}\right)$ de las muestras de papaya, durante el almacenamiento congelado. En el tiempo cero (muestras no congeladas), los tratamientos D06530 y DO6590 no reflejaron cambios significativos ( $>0,05)$ de $h^{\circ}$ respecto a las MNT. Esto indica, que no se presentó un pardeamiento o degradación significativa de los pigmentos naturales de la papaya (Carotenoides), durante la DO, probablemente, a la moderada temperatura $\left(26 \pm 0,2^{\circ} \mathrm{C}\right)$ del proceso osmótico (Talens et al. 2001), mientras que durante el tiempo de almacenamiento, las MNT presentaron significativamente $(\mathrm{p}<0,05)$ valores más altos de ${ }^{\circ}$ (mayores cambios), respecto a los tratamientos osmóticos DO6530 y DO6590. Este resultado indica que las MNT mostraron mayores cambios en su tono por efecto de la congelación, mostrando un desplazamiento desde un tono naranja (natural de la papaya) hacia un tono amarillo. De otra manera, se puede decir que las muestras de papaya tratadas osmóticamente conservan su tono naranja, al ser sometida al almacenamiento en congelación. 


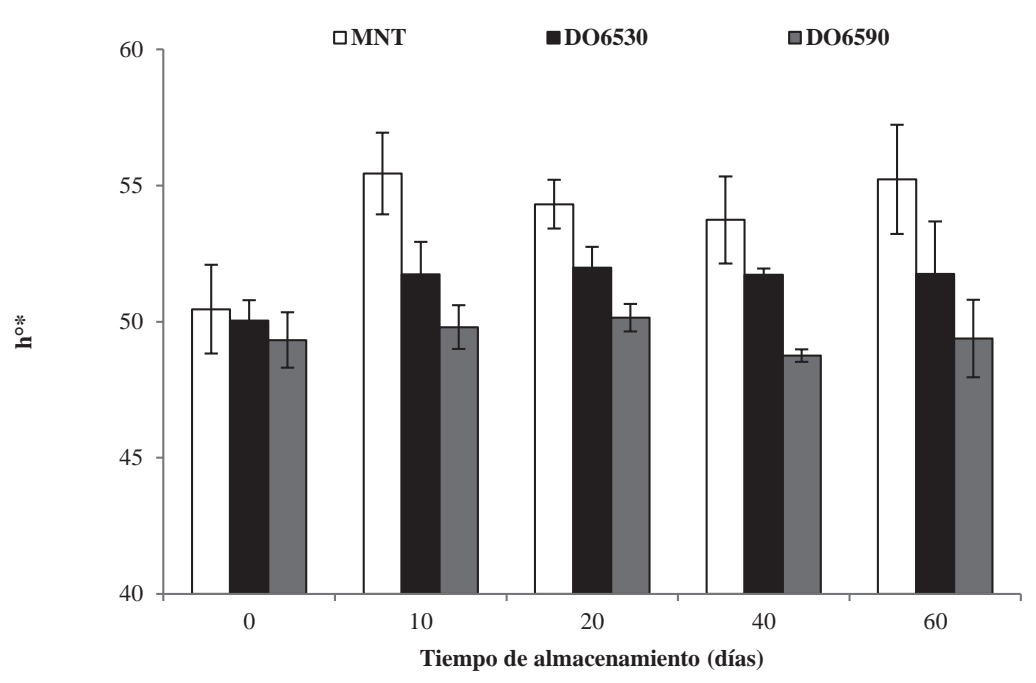

Figura 4. Cambio de tonalidad en muestras de papaya tratadas y no tratadas osmóticamente, durante el almacenamiento en congelación

Con respecto a los dos tratamientos osmóticos, se evidenció que el tratamiento DO6590 mostró valores de $h^{\circ}$ ligeramente menores respecto a DO6530, durante el almacenamiento en congelación; sin embargo, el ANOVA no mostró diferencias estadísticas significativas $(\mathrm{p}>0,05)$.
El cambio total de color $(\Delta \mathrm{E})$, presentado en la figura 5 , muestra en todos los tratamientos un incremento de $\Delta \mathrm{E}$ durante el tiempo de almacenamiento. Este comportamiento, se debe, principalmente, por los cambios de $\mathrm{L}^{*}$, explicados con anterioridad, ya que todos los tratamientos mostraron mayor variación de L*, durante el almace-

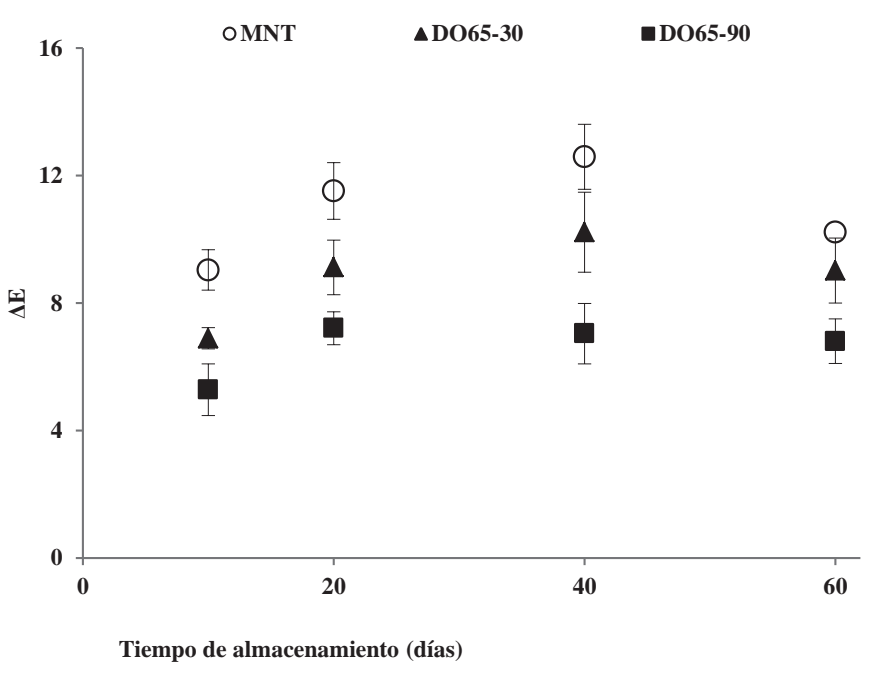

Figura 5. Cambio total de color en muestras de papaya tratadas y no tratadas osmóticamente, durante el almacenamiento en congelación 
namiento, respecto a los cambios del parámetro h. Este resultado corrobora que las muestras no presentaron cambios distintos de la tonalidad naranja de la papaya, resultado importante para la calidad física de la papaya.

Al comparar los dos tratamientos osmóticos y la MNT, se observa que los tratamientos DO6530 y DO6590 presentaron significativamente $(\mathrm{p}<0,05)$ valores inferiores de $\Delta \mathrm{E}$ respecto a las MNT, siendo las muestras del tratamiento DO6590 las que mostraron significativamente $(p<0,05)$ los menores valores (menores cambios de color). Este comportamiento corrobora que el tratamiento MNT presentan los mayores cambios de color frente a los tratamientos osmóticos.

Este efecto positivo de las muestras tratadas osmóticamente puede ser explicado, por un lado, por contener menor agua congelable respecto a la MNT, que contribuye a disminuir las reacciones de pardeamiento en el tejido de la fruta (Wu et al. 2009) y, por otro, a la presencia de azúcar en la superficie de las muestras tratadas, que inhiben la transferencia de oxígeno a la fruta y, por lo tanto, reducen el pardeamiento enzimático (Lenart, 1996; Krokida \& Maroulis, 2001). Comportamiento similar, se encontraron en la osmocongelación de la berenjena (Wu et al. 2009), kiwi y manzana (Marani et al. 2007).

De acuerdo con los resultados de este trabajo, se demuestra que la aplicación de la técnica de osmocongelación es efectiva para disminuir pérdidas de calidad a muestras de papaya en términos de reducción de la PFL, V y color. La mayor efectividad de esta técnica, se presenta al congelar la fruta tratada osmóticamente, con un nivel de contenido de agua de $76,24 \pm 0,41 \%$ (b.h), debido a su menor contenido de agua congelable. El incremento del tiempo de almacenamiento en congelación influyó en la pérdida de calidad de la fruta, mostrando mayor PFL, reducción de volumen y disminución de la claridad.

Conflicto de intereses: El trabajo fue preparado y revisado por el primer autor, con el aval de los otros autores; no existe ningún conflicto de intereses que pongan en riesgo la validez de los resultados presentados. Financiación: Esta Investigación fue financiada por la Escuela de Ingeniería de Alimentos, de la Universidad del Valle.

\section{BIBLIOGRAFÍA}

1. AOAC. 1990. Métodos de análisis de la asociación oficial de química analítica para determinar humedad, fibra, cenizas, grasa y proteína. Washington, U.S.A. Cap. 32, p.1-14.
2. AYALA, A.; CADENA, M.I. 2014. The influence of osmotic pretreatments on melon (Cucumis melo L.) quality during frozen storage. DYNA (Colombia). 81(186): 81-86.

3. AYALA, A.; SERNA, L.; GIRALDO, C. 2009. Efecto de la agitación sobre la deshidratación osmótica de pitahaya amarilla (Selenicereus megalanthus S.) empleando soluciones de sacarosa. Interciencia. (Venezuela). 34(7):492-496.

4. AYSE, İ.; İNCI, T. 2009. Osmotic dehydration of apricot: Kinetics and the effect of process parameters. Chem. Eng. Res. Design (Estados Unidos). 87(2):166-180.

5. BERMÚDEZ, A.; NARVAEZ, M.; CADENA, M.; AYALA, A. 2013. Reducción de pérdida de calidad de melón (Cucumis melo) durante la congelación mediante aplicación previa de deshidratación osmótica. Rev. Alimentos Hoy. (Colombia). 22(30):23-29.

6. BIANCHI, M.; GUAMASCHELLI, A.; MILISENDA, P. 2011. Dehidrocongelación de frutas: Estudio de los parámetros de calidad. Invenio (Argentina). 14(26):117-132.

7. CHIRALT, A.; MARTINEZ, N.; MARTINEZ, J.; TALENS, P.; MORAGA, G.; AYALA, A.; FITO, P. 2001. Changes in mechanical properties throughout osmotic processes: Cryoprotectant effect. J. Food Eng. (Estados Unidos). 49(2-3):129-135.

8. FALADE, K.; IGBEKA, J. 2007. Osmotic Deshydration of Tropical Fruits and Vegetables. Foods Reviews Int. (Estados Unidos). 23:23-373.

9. GONCALVES, E.; ABREU, M.; BRANDAO, T.; SILVA, C. 2011. Degradation kinetics of colour, vitamin $C$ and drip loss in frozen broccoli (Brassica oleracea L. ssp.) during storage at isothermal and nonisothermal conditions. Int. J. Refrigeration (Estados Unidos). 34(8):2136-2144.

10. JAMES, C.; PURNELL, G.; JAMES, J. 2014. Critical Review of Dehydrofreezing of Fruits and Vegetables. Food Bioprocess Technol. (Estados Unidos). 7:1219-1234.

11. KOÇ, B.; EREN, I.; KAYMAK, E. 2008. Modelling bulk density, porosity and shrinkage of quince during drying: The effect of drying method. J. Food Eng. 85(3):340-349. 
12. KROKIDA, M.; MAROULIS, Z. 2001. Quality changes during drying of foods materials. Drying Technol in Agricultural and Food Sciences. Ed. Mujumdar (India). 61p.

13. KUROZAWA, l.; TERNG, I.; DUPAS, M.; JIN, K. 2014. Ascorbic acid degradation of papaya during drying: Effect of process conditions and glass transition phenomenon. J. Food Eng. 123:157-164.

14. LENART, A. 1996. Osmo-convective drying of fruits and vegetables: Technology and application. Drying Technol. (Estados Unidos). 14(2):391-413.

15. MARANI, C.; AGNELLI, M., MASCHERONI, R. 2007. Osmo-frozenfruits: mass transfer and quality evaluation. J. Food Eng. 79(4):1122-1130.

16. MAYOR, L.; CUNHA, R.; SERENO, A. 2007. Relation between mechanical properties and structural changes during osmotic dehydration of pumpkin. Food Res. Int. (Canadá). 40 (4): 448-460.

17. MORAGA, G.; MARTINEZ, N.; CHIRALT, A. 2006. Compositional changes of strawberry due to dehydration, cold storage and freezing-thawing processes. J. Food Proc. Preserv. (Estados Unidos). 30(4):458-474.

18. MOYANO, P.C.; VEGA, R.E.; BUNGER, A.; GARRETÓN, J.; OSORIO, F.A. 2002. Effect of combined processes of osmotic dehydration and freezing on papaya preservation. Food Sci. Technol. Int. (España). 8(5):295-301.

19. OCORÓ, M.; AYALA, A. 2013. Influence of thickness on the drying of papaya puree (Carica papaya L.) through refractance window Technology. DYNA. 182:147-154.

20. OSORIO, C.; FRANCO, M.; CASTAÑO, M.; GONZALEZ, L.; HEREDIA, J.; MORALES, A. 2007. Color and flavor changes during osmotic dehydration of fruits. Innov. Food Sci. Emerg.Technol. (Estados Unidos). 8:353-359.
21. RÓZEK, A.; ACHAERANDIO, I.; GUELL, C.; LOPEZ, F.; FERRANDO, M. 2009. Grape phenolic impregnation by osmotic treatment: Influence of osmotic agent on mass transfer and product characteristics. J. Food Eng. 94(1):59-68.

22. SIMANDJUNTAK, V.; BARRETT, D.; WROLSTAD, R. 1996. Cultivar and frozen storage effects on muskmelon (Cucumis melo), colour, texture and cell wall polysaccharide composition. J. Sci. Food Agric. (Estados Unidos). 71: 291-296.

23. SRIWIMON, W.; BOONSUPTHIP, W. 2011. Utilization of partially ripe mangoes for freezing preservation by impregnation of mango juice and sugars. Food Sci. and Technol. (LWT) (Alemania). 44(2):375383.

24. TALENS, P.; ESCRICHE, I.; MARTINEZ, N.; CHIRALT, A. 2003. Influence of osmotic dehydration and freezing on the volatile profile of kiwi fruit. Food Res. Int. 36(6):635-642.

25. TALENS, P.; MARTINEZ, N.; FITO, P.; CHIRALT, A. 2001. Changes in optical and mechanical properties during osmodehydrofreezing of kiwi fruit. Food Sci. Emerg.Technol. 3(2):191-199.

26. TREGUNNO, N.; GOFF, H. 1996. Osmodehy-drofreezing of apples: structural and textural effects. Food Res. Int. 29(5-6):471-479.

27. WALL, M. 2006. Ascorbic acid, vitamin A, and mineral composition of banana (Musa sp.) and papaya (Carica papaya) cultivars grown in Hawaii. J. Food Compos. Analysis (Estados Unidos) 19(5):434445.

28. WU, L.; ORIKASA, T.; TOKUYASU, K.; SHIINA, T.; TAGAWA, A. 2009. Applicability of vacuum-dehydrofreezing technique for the long-term preservation of fresh-cut eggplant: Effects of process conditions on the quality attribute of the samples. J. Food Eng. 91(4):560-565.

Recibido: Junio 4 de 2014

Aceptado: Septiembre 5 de 2014

Como citar:

Ayala Aponte, A.; Sánchez, M.; Rodríguez, H. 2014. Influencia de la osmocongelación sobre algunas propiedades físicas de papaya (Carica papaya L.). Rev. U.D.C.A Act. \& Div. Cient. 17(2): 487-494. 


\title{
PROPIEDADES REOLÓGICAS DE LA PULPA EDULCORADA DE TAMARINDO
}

\section{RHEOLOGICAL PROPERTIES OF SWEETENED TAMARIND PULP}

\author{
Diego Tirado ${ }^{1}$, Diofanor Acevedo $^{2}$, Piedad Montero ${ }^{3}$
}

\begin{abstract}
${ }^{1}$ Ing. Alimentos. M.Sc. (c). Ingeniería Ambiental. Universidad de Cartagena, Facultad de Ingeniería, Campus de Piedra de Bolívar. Cartagena, Bolívar-Colombia, e-mail: dtiradoa@unicartagena.edu.co; ${ }^{2}$ Ing. Alimentos. Ph.D. Ingeniería de Alimentos. Universidad de Cartagena, Facultad de Ingeniería, Programa de Ingeniería de Alimentos. Avenida El Consulado, calle 30 No, 48-152, Cartagena, Bolívar-Colombia, e-mail: diofanor3000@gmail.com; ${ }^{3}$ Ingeniera de Alimentos. M.Sc., Ph.D (c). Ciencia y Tecnología de Alimentos. Universidad de Cartagena, Facultad de Ingeniería, Programa de Ingeniería de Alimentos, Avenida El Consulado, calle 30 No, 48-152, Cartagena, Bolívar-Colombia, e-mail: pmonteroc@unicartagena.edu.co
\end{abstract}

Rev. U.D.C.A Act. \& Div. Cient. 17(2): 495-501, Julio-Diciembre, 2014

\section{RESUMEN}

El tamarindo es una fruta que se puede utilizar para muchos propósitos, entre ellos, la elaboración de pulpas, que se ha empleado para varios fines. A pesar que el conocimiento de las propiedades viscoelásticas es muy útil en el diseño y predicción de la estabilidad de los productos comestibles, éstas no se han estudiado en la pulpa edulcorada de tamarindo, que presenta algunos problemas durante su almacenamiento. Se determinaron los módulos de almacenamiento, de pérdida y de comportamiento de flujo de la pulpa de tamarindo a dos concentraciones de azúcar. El modulo elástico o de almacenamiento es una medida de la dureza del material. La pulpa con mayor contenido de azúcar presentó una mayor viscosidad aparente, debido a que el carácter sólido de la pulpa se incrementa con el aumento de la proporción de sacarosa y disminuye con el acrecentamiento del porcentaje de pulpa. El módulo elástico fue mayor que el viscoso, demostrando un carácter sólido y más alto para la pulpa con mayor contenido de azúcar.

Palabras clave: Tamarindus indica L., módulo elástico, módulo viscoso, comportamiento de flujo.

\section{SUMMARY}

Tamarind is a fruit that can be used for many purposes, including the manufacture of pulp, which has been employed for various objectives. Although knowledge of the viscoelastic properties is very useful in the design and prediction of the stability of food products, these have not been studied in sweetened tamarind pulp, which presents some problems during storage. Storage modules, loss and flow behavior of tamarind pulp at two sugar concentrations were determined. The elastic or storage modulus is a measure of the hardness of the material. The pulp with higher sugar contents showed a higher apparent viscosity due to the increase of the solid nature of the pulp with the increases in sucrose content, and decrease with increasing percentage of pulp. The elastic modulus was higher than the viscous one, demonstrating strong character, and higher for the pulp with higher sugar content.

Key words: Tamarindus indica L., elastic modulus, viscous modulus, flow behavior.

\section{INTRODUCCIÓN}

El tamarindo (Tamarindus indica L.), pertenece a la familia de las leguminosas y es un árbol nativo del trópico. El fruto es una vaina curvada, constituido por una cáscara de color café brillante y semillas ovaladas aplanadas, unidas entre sí por fibras (Acevedo et al. 2014a; Obulesu \& Bhattacharya, 2011). Es originario del continente africano y fue introducido al continente americano por los españoles. Es un frutal altamente rústico, ya que puede prosperar en suelos pobres o marginados, con poco o nada de riego y cuidados mínimos, con relación a otros frutales tropicales (Viveros et al. 2012). El fruto es una vaina de $12 \mathrm{~cm}$ a $15 \mathrm{~cm}$ de longitud, del cual, la pulpa constituye del 30 al 55\%, la cáscara y la fibra del 11 al 30\% y las semillas, de color marrón, del 33 al $44 \%$. La cosecha, se realiza cuando el fruto alcanza su madurez 
fisiológica, es decir, se deshidrata, pierde peso y adquiere un color gris pardo o marrón y un sonido hueco, cuando las semillas se chocan entre sí. Asimismo, la cáscara del fruto se torna quebradiza cuando se presiona ligeramente con los dedos (Viveros et al. 2012).

Por otro lado, el tamarindo es una fruta versátil, que se puede utilizar para muchos propósitos, entre ellos, la elaboración de pulpas para fines medicinales, aprovechada por diferentes personas en África, Asia y América (Acevedo et al. 2014a; Razali et al. 2012). Un estudio en animales, maniobrando hámsteres, demostró el efecto hipolipemiante de las semillas de tamarindo (Razali et al. 2012). Las frutas y las semillas de esta planta tuvieron un efecto antibacteriano, antinflamatorio y antidiabetogénicos (Razali et al. 2012). En Colombia, una crema hecha de la pulpa de tamarindo, de mantequilla y de otros ingredientes, se dispone para eliminar parásitos de los animales domésticos (Paula et al. 2009).

En muchas áreas tropicales y subtropicales del mundo, el tamarindo se explota para la producción de fruta y, como árbol de sombra (ornamental), en carreteras, en avenidas, en jardines y en parques. En el continente Americano existe en México, Estados Unidos (sur y centro de Florida), Guatemala, Belice, Honduras, Costa Rica, Panamá, norte de Brasil, Colombia, Venezuela y Ecuador. En el Caribe, se reporta su existencia en Cuba, Jamaica, República Dominicana, Haití, Puerto Rico y algunas islas de las Antillas Menores (Bahamas, Barbados e islas Vírgenes). En el continente Asiático, su cultivo se ha extendido a la India, Filipinas, Vietnam, Pakistán, Tailandia, Camboya, Malasia, Indonesia, Laos, Bangladesh. En el medio oriente, se cultiva en Irán y en Arabia Saudita; en África existe en Madagascar, Isla Reunión, Sudán, Senegal, ZimbaGwe, Egipto, Burkinia Faso, Mauritus, Guinea, Níger y Nigeria, entre otros. Además, concurre en el norte de Australia, islas Fiji e Islas Hawaianas (Orozco-Santos, 2001).

El objetivo principal de la explotación del cultivo del tamarindo es la producción de pulpa para su uso en la elaboración de dulces, jugos, jaleas, pulpas edulcoradas, refrescos embotellados, helados y salsas (Ahmed et al. 2007). Se cree que la pulpa sirve para mejorar el apetito y se utiliza para hacer gárgaras para el dolor de garganta y cubrir heridas. Se dice que ayuda a la restauración de la sensibilidad, en casos de parálisis (Razali et al. 2012). El sabor único de dulce y amargo en función de la madurez de la pulpa es muy popular en la cocina mundial (Razali et al. 2012).

Conviene destacar que, aunque la pulpa de tamarindo se ha usado para varios fines, sus propiedades viscoelásticas no se han estudiado en la pulpa edulcorada; estos estudios son necesarios para evaluar algunos problemas durante su almacenamiento y para el diseño y predicción de la estabilidad de la misma.
Por consiguiente, en los ensayos de reología dinámica, se aplica, de forma oscilatoria, una pequeña deformación o velocidad de deformación sobre un fluido y se mide la amplitud de la respuesta del esfuerzo cortante y del ángulo de fase, entre el esfuerzo cortante y la deformación. Este tipo de ensayo permite determinar la proporción entre el componente elástico y viscoso de un material y cuantificar en qué medida se comporta como sólido o como líquido, a través de funciones viscoelásticas, tales como el módulo complejo G* (Acevedo et al. 2014b).

$$
G^{*}=\sqrt{G^{\prime 2}+G^{\prime 2}}
$$

Donde $G^{\prime}$ es el módulo de almacenamiento y representa la componente elástica del producto y $\mathrm{G}^{\prime \prime}$ es el módulo de pérdidas y representa su carácter viscoso (Acevedo et al. 2014b).

$$
\begin{aligned}
& G^{\prime}=\left(\frac{\tau_{0}}{\gamma_{0}}\right) \cos \delta \\
& G^{\prime \prime}=\left(\frac{\tau_{0}}{\gamma_{0}}\right) \operatorname{sen} \delta
\end{aligned}
$$

Donde $\tau_{0}$ y $\gamma_{0}$ son, respectivamente, las amplitudes de las ondas del esfuerzo y de la deformación y $\delta$ es el ángulo de desfasaje. Si el fluido es puramente elástico, $\delta=0^{\circ}$ y $\mathrm{G}^{*}=\mathrm{G}^{\prime}$, $\mathrm{G}^{\prime \prime}=0$; en un fluido viscoso ideal $\delta=90^{\circ}$ y $\mathrm{G}^{\prime \prime}=\mathrm{G}^{*}, \mathrm{G}^{\prime}=0$ (Acevedo et al. 2014b).

Las medidas reológicas se han considerado como una herramienta analítica para proporcionar conocimientos fundamentales sobre la organización estructural de alimentos (Acevedo et al. 2014b; Ahmed et al. 2007). Estas son importantes en la formulación, el procesamiento, el transporte y el almacenamiento, especialmente, para emulsiones y suspensiones. El conocimiento de las propiedades viscoelásticas es muy útil en el diseño y la predicción de la estabilidad de muestras almacenadas (Ramos \& Ibarz, 1998). Uno de los métodos frecuentemente usado para caracterizar la viscoelasticidad de los fluidos alimenticios son los ensayos de reología dinámica, en el cual, en este tipo de ensayos, se aplica, de forma oscilatoria, una pequeña deformación o velocidad de deformación sobre un fluido midiendo la amplitud de la respuesta del esfuerzo cortante y del ángulo de fase, entre el esfuerzo cortante y la deformación (Acevedo et al. 2014b). Este ensayo corresponderá al estado de viscoelasticidad lineal si el esfuerzo es linealmente proporcional a la deformación aplicada y si la respuesta del esfuerzo es en forma de una onda sinusoidal; en el caso de un sólido elástico, esta onda está en fase con la velocidad de deformación 
aplicada. Para un líquido viscoso ideal existe un desfase de $90^{\circ}$ entre ellos; en fluidos viscoelásticos, el ángulo de desfase está comprendido entre 0 y $90^{\circ}$ (Acevedo et al. 2014b).

El objetivo de la presente investigación fue evaluar las propiedades reológicas de la pulpa edulcorada de tamarindo ( $T$. indica L.), a diferentes concentraciones de pulpa.

\section{MATERIALES Y MÉTODOS}

Elaboración de la pulpa. Se obtuvieron frutos de tamarindo (T. indica L.) en estado de madurez comercial, sanos, libres del ataque de hongos y gorgojos, a los cuales, se retiró la cáscara y la semilla. En un recipiente, se colocaron $4 \mathrm{Kg}$ de semillas y se le agregaron $7 \mathrm{Kg}$ de agua potable, se mezcló vigorosamente con un agitador y se dejó en reposo durante 3 horas. Se eliminó el agua de lavado y, a la parte sólida, se le realizó despulpado, con una malla de 0,06 pulgadas. La pulpa obtenida con $10^{\circ}$ Brix, se mezcló en una relación 6:4 y 7:3 de pulpa a azúcar, respectivamente. La pulpa, se empacó y se selló en bolsas de polietileno de alta densidad; antes de sellar, se eliminó el aire atrapado dentro de la bolsa y se dejó un borde libre de $1,5 \mathrm{~cm}$, aproximadamente.

Análisis fisicoquímico. El contenido proximal a la pulpa se efectuó, según los métodos de la A.O.A.C. (2005); la humedad, se determinó, según método 925.09b: desecación a $105^{\circ} \mathrm{C}$, hasta peso constante; la proteína, según método 960.52: Kjeldahl, tratamiento con ácido sulfúrico concentrado; la grasa, según método 920.39: Soxhlet, con extracción discontinua con éter de petróleo y, luego de la evaporación del solvente, se registró el peso del extracto etéreo (grasa bruta); las cenizas, según método 923.03: Por calcinación a $550^{\circ} \mathrm{C}$, hasta la obtención de cenizas blancas; fibra dietaría total, según 991.43: Método enzimático-gravimétrico, con amilasas, proteasa y amilogluco-sidasa, se determina la fibra dietaria, como el residuo indigerible por los sucesivos tratamientos enzimáticos y, finalmente, los carbohidratos se determinaron por diferencia (se resta de 100 la suma de todos los macronutrientes, incluida la fibra dietaría y la humedad).

Análisis microbiológico. Para la determinación de la calidad microbiológica, se siguió lo indicado por la Normas Técnicas Colombiana (NTC) 5468, propuesta por El Instituto Colombiano de Normas Técnicas y Certificación (ICONTEC), para establecer los requisitos y los métodos de ensayo que deben cumplir los jugos (zumos), las pulpas, los néctares de frutas y sus concentrados, para consumo directo o elaboración posterior. La determinación de microorganismos mesófilos, se realizaron según NTC 4519; coliformes totales y fecales, según NTC 4458; esporas del Clostridium spp. y bacterias sulfito reductoras, según NTC 4834 y, hongos y levaduras, según NTC 4132. Las pruebas se realizaron por triplicado.
Determinaciones reológicas. Se tomaron tres muestras de $500 \mathrm{~g}$ de pulpa y se evaluaron $1 \mathrm{~g}$ en un reómetro. Las medidas viscoelásticas, se efectuaron en un reómetro TA AR $1500^{\circledR}$ (TA Instruments Ltda.), utilizando la geometría de platos concéntricos (40mm steel plate). Previo a la medición, todas las muestras se dejaron en reposo durante 15 minutos, para permitir la relajación de las mismas. A cada una de las muestras, se les realizaron barridos de deformación (strains weep), para elegir el valor de la deformación y determinar el rango de viscoelásticidad lineal y proceder a ejecutar el barrido de frecuencia (frequency sweep), en un rango entre $0,1 \mathrm{~Hz}$ a $10 \mathrm{~Hz}$. La temperatura de las muestras, se mantuvo a $25,0 \pm 0,1^{\circ} \mathrm{C}$; la entrada de la deformación y la salida de los datos de esfuerzo cortante fue automáticamente controlado por el programa TA Universal Analysis Versión $5.2^{\circledR}$, instalado en un ordenador PC, conectado al reómetro; el análisis de datos reológicos, se realizó utilizando el software Rheology Advantage Data Analysis Version 5.7 ${ }^{\circledR}$. Los resultados de los ensayos reológicos experimentales oscilatorios, se expresan en términos del módulo de almacenamiento $\left(G^{\prime}\right)$ y del módulo de pérdida (G'), en función de la frecuencia de la deformación. A la pulpa colocada en el reómetro, se le realizó un ciclo de deformación de $0,0716 \mathrm{~S}^{-1}$ a $100 \mathrm{~S}^{-1}$ en 60 segundos.

Diseño de experimento. En la investigación, se manejó un diseño experimental totalmente aleatorio. Las determinaciones, se efectuaron por triplicado y los resultados expresados como la media \pm la desviación estándar. Se utilizó el programa estadístico Statgraphics Centurion XVI.I; se calcularon la media y la desviación estándar de los resultados en los análisis efectuados. Para el análisis de los datos, se aplicó la prueba t-student; se fijó el nivel de significancia a $p<0,05$; las variables respuestas fueron las características fisicoquímicas (humedad, proteína, grasa, carbohidratos, ceniza y fibra); la calidad microbiológica (Mesófilos, Coliformes totales y fecales, esporas del Clostridium spp., bacterias sulfito reductoras, hongos y levaduras) y las características reológicas: Viscosidad aparente, módulo de almacenamiento y pérdida.

\section{RESULTADOS Y DISCUSIÓN}

Análisis fisicoquímicos. En la tabla 1, se muestra la composición proximal de la pulpa sin azúcar ( $100 \%$ pulpa) y pulpas con relaciones de pulpa-azúcar 6:4 (60\% pulpa) y 7:3 (70\% pulpa), las cuales, presentan diferencias significativas $(p<0,05)$. Las diferencias entre una mezcla de pulpa y otra, se deben a los diferentes contenidos de azúcares que cada uno presenta (Moreno et al. 2003).

El porcentaje de humedad de las pulpas es bajo, comparado con la mayoría de las frutas tropicales, que tienen valores entre 80 y 90\% (Hincapié et al. 2012). 
Tabla 1. Composición proximal para muestras con $60 \%$, $70 \%$ y $100 \%$ de pulpa de tamarindo.

\begin{tabular}{|c|c|c|c|}
\hline \multirow{2}{*}{ Componente (\%) } & \multicolumn{3}{|c|}{ pulpa } \\
\cline { 2 - 4 } & $100 \%$ & $70 \%$ & $60 \%$ \\
\hline Humedad & $50 \mp 2,34$ & $40 \mp 0,95$ & $35 \mp 1,57$ \\
\hline Proteína & $1,2 \mp 0,06$ & $0,94 \mp 0,02$ & $0,89 \mp 0,04$ \\
\hline Grasa & $0,97 \mp 0,03$ & $0,83 \mp 0,05$ & $0,25 \mp 0,01$ \\
\hline Cenizas & $1,3 \mp 0,06$ & $1,40 \mp 0,02$ & $1,83 \mp 0,93$ \\
\hline Fibra & $1,7 \mp 0,07$ & $1,1 \mp 0,07$ & $1,03 \mp 0,67$ \\
\hline Carbohidratos & $44 \mp 1,23$ & $55,66 \mp 1,56$ & $61 \mp 1,85$ \\
\hline
\end{tabular}

Análisis microbiológicos. En la tabla 2, se muestran los resultados de los análisis microbiológicos para las pulpas. Éstas se encuentran dentro del rango de aceptabilidad, según la NTC 5468, en donde se halla el índice máximo permisible para identificar el nivel de buena calidad microbiológica de pulpa de frutas, tal como se observa en la tabla 2. Resultados cercanos, se presentaron en el recuento microbiano de pulpa de guanábana (Annona muricata L.), que realizaron Ramírez-Méndez et al. (2009). Hincapié et al. (2012), en la elaboración de una bebida energizante a partir de borojó (Borojo apatinoi Cuatrec.), encontró, igualmente, que la pulpa del fruto se localiza dentro de los parámetros establecidos por la NTC 5468.
Comportamiento de flujo. La viscosidad aparente en función de la velocidad de corte de las pulpas con 30\% y $40 \%$ de azúcar (70\% y $60 \%$ de pulpa, respectivamente), se muestra en la figura 1 . Allí, se observa que a medida que aumenta la velocidad de corte, independientemente del porcentaje de pulpa, la viscosidad aparente también disminuye. Ahmed et al. (2007), en su investigación, estudiaron las propiedades reológicas de tamarindo concentrado, en un rango de temperaturas de $10^{\circ} \mathrm{C}$ a $90^{\circ} \mathrm{C}$, usando un reómetro de esfuerzo controlado, registrando resultados similares a los de esta investigación. De acuerdo a la figura 1 , se puede concluir que la pulpa con mayor contenido de azúcar (40\%) presenta una mayor viscosidad aparente, debido a que el carácter sólido

Tabla 2. Recuento microbiano para muestras con $60 \%, 70 \%$ y $100 \%$ de pulpa.

\begin{tabular}{|c|c|c|c|c|}
\hline \multirow{2}{*}{ Microorganismo (UFC/g) } & \multicolumn{3}{|c|}{ pulpa } & Permitido según \\
NTC 5468
\end{tabular}

de la pulpa se incrementa con el aumento de la proporción de sacarosa y disminuye con el aumento del porcentaje de pulpa, porque la sacarosa liga los componentes de la pulpa haciendo que ésta oponga más resistencia al flujo (Acevedo et al. 2013).
Pruebas dinámicas oscilatorias. En la figura 2, se puede observar que, a medida que aumenta la frecuencia, el módulo de almacenamiento $\left(\mathrm{G}^{\prime}\right)$ de la pulpa con $60 \%$ es mayor que el módulo de almacenamiento $\left(\mathrm{G}^{\prime}\right)$ de la pulpa con $70 \%$. Por el contrario, el módulo de pérdida (G") de la pulpa con 


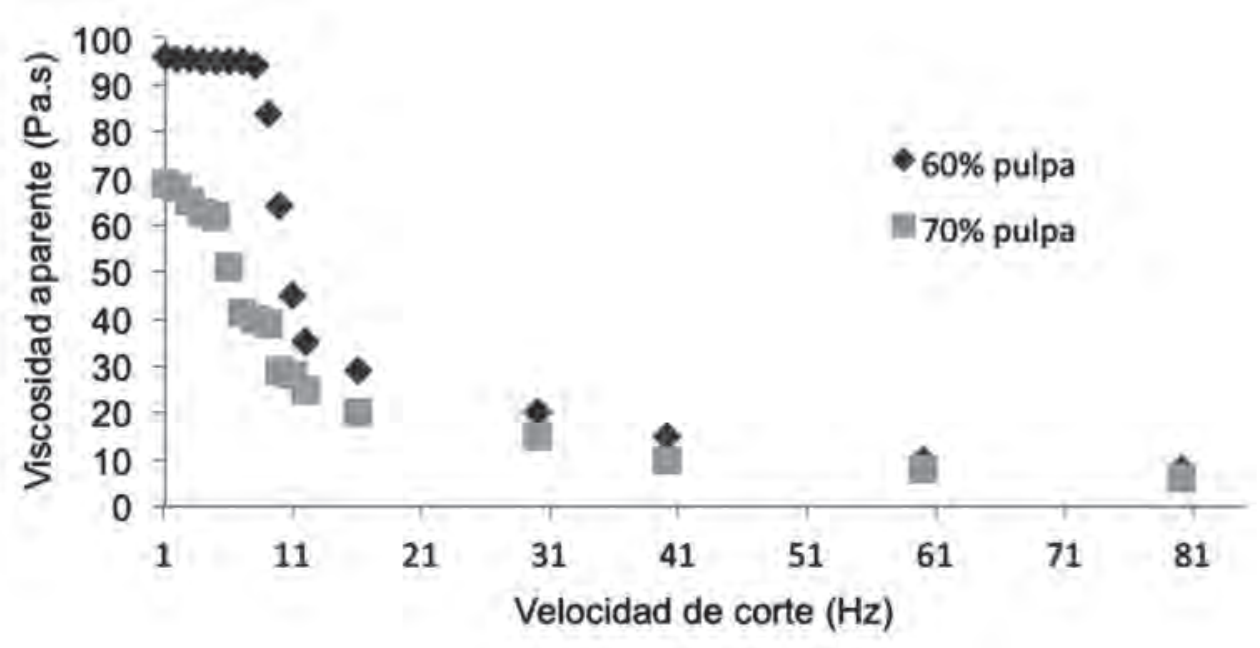

Figura 1. Viscosidad aparente en muestras con $60 \%$ y $70 \%$ de pulpa de tamarindo.

el $60 \%$ fue menor que él módulo de pérdida (G”) de la pulpa con el $70 \%$, llegando un instante a $10 \mathrm{~Hz}$ en el que se igualaron los módulos. También, se puede mostrar, que el modulo elástico $\left(G^{\prime}\right)$ es mayor que él modulo viscoso ( $\left.G^{\prime \prime}\right)$ en todas las repeticiones que se realizaron a las dos concentraciones de pulpa, mostrando un comportamiento elástico en el que ( $\left.\mathrm{G}^{\prime}\right)$ de la pulpa con $60 \%$ tendió a igualarse con el (G"), con $70 \%$ de pulpa, comportándose de una manera elástico-viscoso. En estudios similares, Magaña-Barajas et al. (2009) evaluaron la caracterización viscoelástica de masas de variedades de trigos suaves, en el cual, se evidenció que el G' y G' permaneció constante respecto al tiempo, con una frecuencia de $5 \mathrm{rad} / \mathrm{s}$ y $10 \mathrm{rad} / \mathrm{s}$; también los parámetros $\mathrm{G}^{\prime}$ y G" representaron un comportamiento elástico y viscoso.
En otros trabajos Ramos \& Ibarz (2006), caracterizaron las propiedades viscoelásticas de muestras de pulpa de membrillo, en el rango de concentraciones de 12,3 a $28^{\circ}$ Brix, a la temperatura de $20^{\circ} \mathrm{C}$. Sus resultados demostraron que, para la muestra de $28^{\circ} \mathrm{Brix}$, la pulpa se comportó como un semi-sólido, en todo el rango de frecuencia estudiada. Para la concentración de $24^{\circ}$ Brix, a bajas frecuencias, la pulpa se comportó como un semi-líquido (G">G'), mientras que en frecuencias más altas, se comportó como semi-sólido (G'> G'). Para la pulpa de $16^{\circ}$ Brix y $20^{\circ}$ Brix hubo el predominio del carácter viscoso sobre el carácter elástico; para la pulpa de $12,3^{\circ}$ Brix, no fue posible determinar los valores G' y G". Los autores concluyeron que la viscosidad compleja de pulpa de membrillo aumenta con la concentración para todo

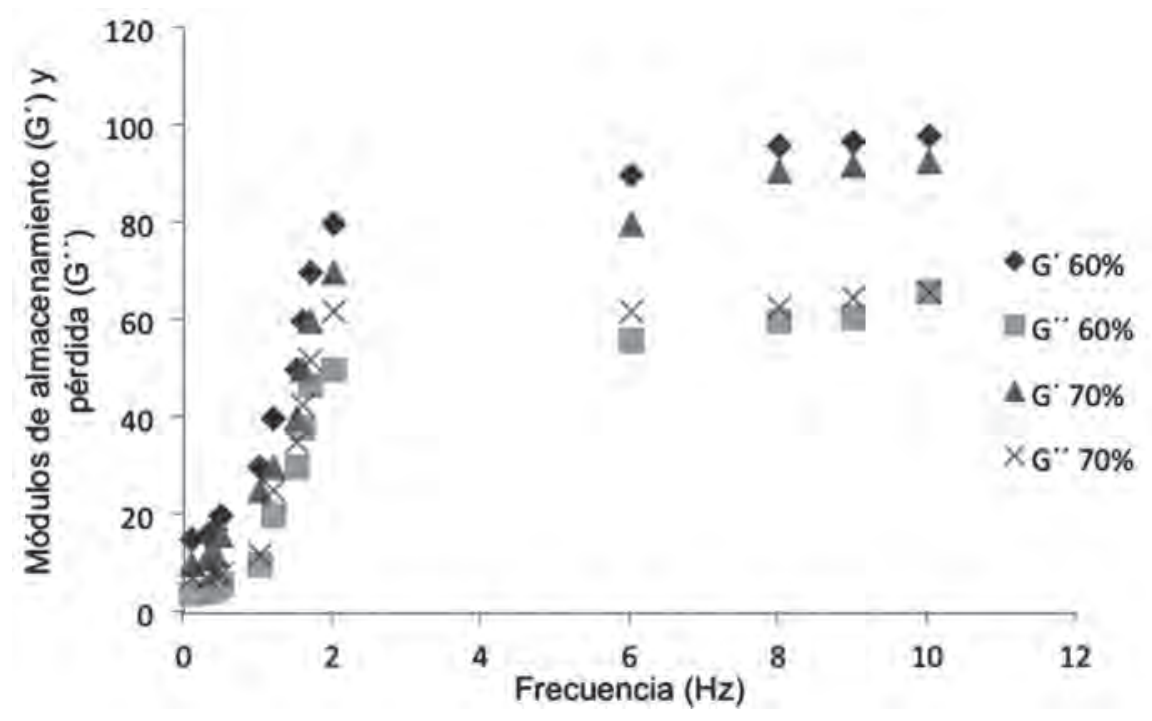

Figura 2. Módulos de almacenamiento $\left(\mathrm{G}^{\prime}\right)$ y pérdida $\left(\mathrm{G}^{\prime \prime}\right)$ de muestra con 60 y $70 \%$ de pulpa de tamarindo. 
el rango de frecuencias estudiadas, al igual que lo expuesto en esta investigación.

Los resultados obtenidos de la caracterización viscoelástica de esta investigación son semejantes a los encontrados en otros trabajos de Ahmed et al. (2007), quienes estudiaron las características reológicas de tamarindo (T. indica L.), en el cual, se observó que, a medida que utilizaban velocidades de corte bajas, la viscosidad no variaba significativamente.

La calidad microbiológica de la pulpa presentó características óptimas, debido a la alta concentración de azúcar que las cubría.

Conflictos de intereses: El manuscrito fue preparado y revisado con la participación de todos los autores, quienes declaramos que no existe conflicto de intereses, que ponga en riesgo la validez de los resultados presentados.

\section{BIBLIOGRAFÍA}

1. A.O.A.C. 2005. Official Methods of Analysis of the AOAC International. 18th ed. Horowitz, W.; Latimer Jr., G.W. (eds) Association of Analytical Chemists. Maryland (United States). 780p.

2. ACEVEDO, D.; GRANADOS, C.; TORRES, R. 2014a. Caracterización reológica del suero costeño de Turbaco, Arjona, El Carmen de Bolívar y uno comercial (Colombia). Información Tecnológica. (Chile). 25(3):3-10.

3. ACEVEDO, D.; TIRADO, D.F.; GUZMÁN, L.E. 2014b. Deshidratación osmótica de pulpa de tamarindo (Tamarindus indica L.): influencia de la temperatura y la concentración. Rev. U.D.C.A. Act. \& Div. Cient. 17(1):123-130.

4. ACEVEDO, D.; RODRIGUEZ, A.; FERNANDEZ, A. 2013. Efectos simultáneos de las variables de proceso sobre las propiedades de flujo del suero costeño. Rev. Biotecnolgía en el Sector Agropecuario y Agroindustrial. (Colombia). Edición Especial (2):112-117.

5. AHMED, J.; RAMASWAMY, H.S.; SASHIDHAR, K.C. 2007. Rheological characteristics of tamarind (Tamarindus indica L.) juice concentrates. LWT-Food Sci. Technol. (United States). 40(2):225-231.

6. MAGAÑA-BARAJAS, E.; RAMÍREZ-WONG, B.; PLATTLUCERO, L. C.; LÓPEZ-AHUMADA, G.A.; TORRES, P.I.; SÁNCHEZ-MACHADO, D.I. 2009. Caracteriza- ción viscoelástica de masas de variedades de trigos suaves. Tecnología, Ciencia, Educación. 24(1):12-22

7. HINCAPIÉ, G.A.; PALACIO, J.C.; PÁEZ, S.; RESTREPO, C.E.; VÉLEZ, L.M. 2012. Elaboración de una bebida energizante a partir de borojó (Borojo apatinoi Cuatrec.). Rev. Lasallista Invest. 9(2):33-43.

8. MORENO, M.J.; GIRÁN, N.; SERRANO, K.; GARCÍA, D.; BELÉN, D.R. 2003. Evaluación microbiológica y fisicoquímica de néctares pasteurizados elaborados con pulpa de tomate de árbol (Cyphomandra betaceae Sendth). ALAN. 53(3):282-286.

9. OBULESU, M.; BHATTACHARYA,S. 2011. Color changes of tamarind (Tamarindus indica L.) pulp during fruit development, ripening, and storage. Int. J. Food Properties. (United Kingdom). 14(2):538-549.

10. OROZCO-SANTOS, M. 2001. El cultivo de tamarindo (Tamarindus indica L.) en el trópico seco de México. Folleto Técnico N¹. Ed. Tecomán, Col. (México). 96p.

11. PAULA, F.S.; KABEYA, L.M.; KANASHIRO, A.; DE FIGUEIREDO, A.S.; AZZOLINI, A.E.; UYEMURA, S.A.; LUCISANO-VALIM, Y.M. 2009. Modulation of human neutrophil oxidative metabolism and degranulation by extract of Tamarindus indica L. fruit pulp. Food Chem. Tox. (United Kingdom). 47:163-170.

12. RAMÍREZ-MÉNDEZ, R.; ACOSTA, K.; ARENAS, L.; YAMARTE, M.; SANDOVAL, L. 2009. Efecto del escaldado sobre la calidad microbiológica de pulpa de guanábana (Annona muricata L.). Boletín Centro Invest. Biológicas (Venezuela). 43(1):97-99.

13. RAMOS, A.M.; IBARZ, A. 1998. Tixotropy of orange concentrate and quince puree. J. Texture Studies. (United Kingdom.). 29:313-324.

14. RAMOS, A.M.; IBARZ, A. 2006. Comportamiento viscoelástico de pulpa de membrillo en función de la concentración de sólidos solubles. Ciência e Tecnologia Alimentos. (Brazil). 26(1):214-219.

15. RAZALI, N.; MAT-JUNIT, S.; ABDUL-MUTHALIB, A.F.; SUBRAMANIAM, S.; ABDUL-AZIZ, A. 2012. Effects of various solvents on the extraction of antioxidant phenolics from the leaves, seeds, veins and skins of Tamarindus indica L. Food Chem. (United Kingdom). 131(2):441-448. 
16. VIVEROS, J.C.; FIGUEROA, K.A.; GALLARDO, F.; GAR- $\quad$ Recibido: Mayo 26 de 2014

CÍA, E.; RUIZ, O.; HERNÁNDEZ, F. 2012. Sistemas Aceptado: Octubre 20 de 2014 de manejo y comercialización de tamarindo (Tamarindus indica L.) en tres municipios de Veracruz. Rev. Mex. Ciencias Agrícolas. 3(6):1217-1230.

Como citar:

Tirado, D.; Acevedo, D.; Montero, P. 2014. Propiedades reológicas de la pulpa edulcorada de tamarindo. Rev. U.D.C.A Act. \& Div. Cient. 17(2): 495-501. 